\title{
Fisiopatología, diagnóstico diferencial y tratamiento de la apnea emotiva grave. A propósito de un caso clínico
}

\author{
MARTA HERNÁNDEZ CH. ${ }^{1}$, NATALIA ESQUIVEL P. ${ }^{2}$, MAURICIO LÓPEZ E.
}

1. Neuropediatra, Residente Neurología Pediátrica División de Pediatría. Unidad Neuropediatría. Facultad de Medicina, Pontificia Universidad Católica de Chile.

2. Residente de Pediatría, Universidad de Antofagasta. Servicio de Pediatría Hospital Clínico Regional de Antofagasta.

\begin{abstract}
Pathophysiology, differential diagnosis and treatment of severe emotional apnea: Based on report case

Introduction: Emotional apneas (EA) are non-epileptic paroxysmal events affecting 5\% of healthy children. The diagnosis is based on a stereotyped sequence of clinical events that start with tears caused by emotional stimulus, resulting in an autonomic nervous system alteration with transient color change, pale or cyanotic. $15 \%$ of the cases are associated with loss of consciousness, changes in tone or tonic-clonic movements secondary to hypoxia. Objective: To report a case of severe EA and to review the differential diagnosis and preventive treatments. Case report: A 15 -month old infant with cyanotic emotional apnea since 8 months of age, triggered by pain, disgust or fear, increasing in frequency (3-4 per day) and intensity with altered consciousness and hypotonia. At 12 months, the patient also presented generalized tonic-clonic seizures of 3 minutes long, reason why the infant was admitted to the emergency service. Normal psychomotor development as well as normal physical, neurological and laboratory test results (without anemia) were found. Electroencephalography and brain MRI presented no abnormalities. Preventive therapy using Piracetam was performed in order to reduce crisis, which occurred in the first month of treatment. Conclusions: In most cases, a timely information delivery to parents is enough due to the benign nature and natural history of EA. However, when the frequency and severity of EA impact the child and family, to rule out heart disease or epilepsy and to seek preventive treatment options are required.
\end{abstract}

(Key words: Emotional apnea, autonomic syncope, paroxysmal event, expiratory apnea, autonomic dysfunction).

Rev Chil Pediatr 2014; 85 (4): 481-485

\section{RESUMEN}

Introducción: Las apneas emotivas (AE) son eventos paroxísticos no epilépticos que afectan al $5 \%$ de niños sanos. El diagnóstico se basa en una secuencia estereotipada de eventos clínicos iniciado con llanto provocado

Recibido el 16 de abril de 2014, última versión aceptada para publicación el 25 de junio de 2014.

Potenciales conflictos de interés: Este trabajo cumple con los requisitos sobre consentimiento /asentimiento informado, comité de ética, financiamiento, estudios animales y sobre la ausencia de conflictos de intereses según corresponda. 
por un estimulo emocional que desencadena una alteración refleja del sistema nervioso autonómico con cambio de color, pálido o cianótico. En el $15 \%$ se asocia a pérdida de conciencia, cambios del tono o movimientos tónico-clónicos secundarios a hipoxia. Objetivo: Presentar un caso clínico de AE grave, revisar el diagnóstico diferencial y tratamientos preventivos. Caso clínico: Lactante de 15 meses con AE cianóticas a partir de los 8 meses de edad, desencadenados por dolor, disgusto o miedo que aumentaron en frecuencia (3-4 por día) e intensidad con alteración de conciencia e hipotonía. A los 12 meses, se agregó además una crisis tónico clónica de 3 min de duración, por la cual ingresó a Servicio de Urgencia. Se constató un desarrollo psicomotor normal, examen físico, neurológico y parámetros de laboratorio normales (sin anemia), al igual que la electroencefalografía y resonancia magnética cerebral. Se inició terapia preventiva con piracetam con el propósito de disminuir las crisis, lo que se produjo desde el primer mes de tratamiento. Conclusiones: En la mayoría de los casos, la naturaleza benigna e historia natural de las AE permiten que una entrega de información oportuna a los padres sea suficiente. Sin embargo, cuando la frecuencia y severidad de las AE impactan al niño y su familia, se requiere descartar patología cardíaca o epilepsia y buscar opciones de tratamiento preventivo.

(Palabras clave: Apnea emotiva, sincope autonómico, evento paroxístico cerebral, apnea espiratoria, disfunción autonómica).

Rev Chil Pediatr 2014; 85 (4): 481-485

\section{Introducción}

Las apneas emotivas (AE) son eventos paroxísticos no epilépticos que afectan aproximadamente al $5 \%$ de los niños sanos ${ }^{1}$, aunque hay autores que creen que su verdadera frecuencia es hasta $27 \%$ en grado leve ${ }^{2}$. El $76 \%$ de los casos ocurre entre los 6 meses y 4 años con un rango de 3 días a 8 años de edad. La frecuencia puede ser variable entre una vez al mes hasta varias crisis al día. La duración del evento es menor a 3 min con recuperación espontánea en la mayoría de los $\operatorname{casos}^{3,4}$.

Las AE ocurren en vigilia y según el cambio de la coloración de la piel se clasifican en $\mathrm{AE}$ pálida, $\mathrm{AE}$ cianótica y $\mathrm{AE}$ mixta. La $\mathrm{AE}$ ocurre en una secuencia estereotipada de eventos clínicos gatillados por circunstancias específicas (dolor, disgusto u otra provocación) que llevan a un llanto con posterior alteración del tono y eventualmente un sincope. Este síncope reflejo puede ser inspiratorio (AE pálida), espiratorio (AE cianótica) o mixto con una proporción de 5:3:25. Puede ir seguido de compromiso de conciencia, secundaria a falla del flujo sanguíneo cerebral y cambios en el tono postural. Las AE graves son aquellas que presentan compromiso de conciencia prolongado, convulsiones o status epilépticus como consecuencia de hipoxia o isquemia cerebral relativa y corresponde al $15 \%$ de todas las $\mathrm{AE}^{6}$.
La disfunción del sistema nervioso autónomo parece jugar un rol fundamental en la AE. La etiopatogenia de la AE pálida sería un reflejo parasimpático con bradicardia, asistolia y sincope asistólico reflejo ${ }^{7-9}$. En cambio en la AE cianótica habría una estimulación simpática con un sincope reflejo de apnea espiratoria ${ }^{10}$, probablemente shunt intrapulmonar, que lleva a una rápida cianosis ${ }^{11}$. De todas maneras una $\mathrm{AE}$ cianótica no excluye la posibilidad de bradicardia y asistolia y puede haber activación consecutiva de ambos sistemas autonómicos en una misma AE. Un niño puede tener los dos tipos de $\mathrm{AE}$, las que igualmente pueden suceder en una misma familia.

Dada la naturaleza benigna y el pronóstico favorable de esta condición, en los casos leves el tratamiento indicado es un asesoramiento que de confianza y tranquilice a los padres. Para los casos graves es necesario descartar diagnósticos diferenciales del área cardiológica (síncope cardiogénico) y neurológica (epilepsias, apneas centrales primarias) e indicar terapias preventivas.

El propósito de este manuscrito es presentar un caso clínico de AE grave y por medio de este, reportar información científica actualizada en relación a su fisiopatología, con énfasis en el diagnóstico diferencial y tratamientos preventivos potenciales de estos eventos. 


\section{Caso clínico}

Niña de 15 meses, previamente sana, tercera hija de matrimonio no consanguíneo, sin antecedentes familiares de epilepsia o AE. A los 8 meses de vida y en relación a un ruido fuerte, presentó un evento descrito por su madre con llanto intenso, persistente, apnea espiratoria, cianosis, desviación de la mirada pérdida de conciencia e hipotonía. Remitió espontáneamente después de unos segundos con una vigilia normal luego de este evento. Estos episodios continuaron haciéndose más frecuentes e intensos, hasta 3-5 por día y seguidos de compromiso de conciencia e hipotonía, por lo que consultó a los 10 meses a neurología donde se realizó un EEG estándar que fue informado como normal.

A los 12 meses y después de una caída en el piso, nuevamente presentó llanto, apnea espiratoria, cianosis seguida de pérdida de conciencia, hipotonía a la que se agregó una crisis tónico clónica generalizada de $3 \mathrm{~min}$ de duración. Ingresó al servicio de urgencia donde se constató lactante vigil, con buen desarrollo psicomotor. Se realizó hemograma que descartó anemia, sin microcitosis, estudio de cinética de fierro normal, electroencefalograma (EEG) normal y resonancia magnética (RM) de cerebro normal. Se dió alta con diagnóstico de apnea emotiva cianótica grave. Se solicitó controles ambulatorios.

Posterior al alta persistió con AE de frecuencia diaria, con intensidades de leves a graves. El estudio de cinética de fierro fue normal, por lo que se inició tratamiento con piracetam dosis $40 \mathrm{mg} / \mathrm{kg}$ día fraccionado en dos dosis por 4 meses. En controles posteriores se pudo verificar disminución de la frecuencia e intensidad de las crisis, con sólo 2 apneas emotivas con leve cianosis y recuperación espontánea.

\section{Discusión}

La frecuencia de las AE en niños sanos y la gravedad de un pequeño porcentaje de ellas, como en nuestro caso, hace necesario conocer la fisiopatología y los posibles tratamientos preventivos necesarios para disminuir el fuerte impacto que éstas provocan en el niño y su familia. El caso descrito corresponde a una $\mathrm{AE}$ cianótica grave con eventos que presentan, aparte del cambio de coloración de la piel, compromiso de conciencia transitorio y convulsión anóxica.

Se ha descrito una mayor prevalencia de $\mathrm{AE}$ en hijos de padres consanguíneos (OR 11,13; CI $4,53-27,37$ ) y hasta en un $20-30 \%$ de familiares de niños con $\mathrm{AE}^{1,5}$, lo que complementaría estudios previos de DiMario y Sarfarazi, que postularon una herencia autosómica dominante con penetrancia reducida ${ }^{1,5,12}$. Considerando lo anterior, se postula que una disfunción autonómica subyacente y genéticamente determinada, jugaría un rol fundamental ${ }^{6,13}$ en ambos tipos de AE (pálida y cianótica).

En la AE pálida, la disfunción del sistema nervioso autónomo parasimpático lleva a bradicardia y asistolia. La función vagal, evaluada mediante diversos test neurofisiológicos, como el reflejo oculocardíaco, el test de pilocarpina y parámetros de variabilidad de la frecuencia cardíaca en Holter de 24 h, han evidenciado una sobre-reactividad, más que sobreactividad vagal $^{14-16}$. Para antagonizar la sobre-reactividad vagal y prevenir la AE se han usado drogas anticolinérgicas como atropina, escopolamina y glicopirrolate ${ }^{17,18}$. También se ha usado teofilina, principalmente por su función inótropa positiva, considerándola en segundo plano como estimuladora de los centros respiratorios bulbares. En casos muy complicados y refractarios al tratamiento médico, se ha indicado la implantación de marcapasos, con buenos resultados $^{8,19}$.

La AE cianótica obedecería a estimulación sistema nervioso autonómo simpático y en su etiopatogenia se ha asociado, en algunos casos, la anemia ferropriva. La disfunción emocional e irritabilidad propia de un niño con anemia ferropriva aumentaría el riesgo de $\mathrm{AE}$, al mismo tiempo que los bajos niveles de hemoglobina disminuirían la capacidad de transportar oxígeno a los tejidos ${ }^{2,6,20}$ favoreciendo la anoxia cerebral y estimulando secreción de eritropoyetina. El mayor consumo de fierro favorecido por la secreción de eritropoyetina disminuiría aún más los depósitos, perpetuando la anemia ${ }^{21}$. Consistente con estos hallaz- 
gos, Oreii et y Zehetner, demostraron que la suplementación con fierro en anemia ferropriva sería efectiva en optimizar la disregulación del sistema nervioso autonómo y prevenir las $\mathrm{AE}^{21,22}$, quedando aún pendiente estudiar este efecto en poblaciones no deficientes ${ }^{17}$. Igualmente, la deficiencia de fierro per se, agrega estrés oxidativo que altera el metabolismo de las catecolaminas y la función de neurotransmisores $^{23}$.

El piracetam (2-oxo-1-pyrrolidine), usado en nuestro paciente, es un derivado cíclico del ácido gamma aminobutírico (GABA) y tendría un efecto sobre el aumento de la oxigenación tisular y aumenta el proceso inhibitorio de hiperpolarización similar al GABA. Ha sido usado en varios desórdenes cognitivos y está aprobada por la FDA para mioclonus no epiléptico. Sawires y cols. ${ }^{24}$ y Abbaskhanian y cols. ${ }^{25}$, en dos estudios randomizados doble ciego, demostraron una reducción de crisis concluyendo que el piracetam con una dosis de $40-60 \mathrm{mg} / \mathrm{kg} /$ día es un tratamiento seguro y efectivo para prevenir AE.

La estructura familiar y la actitud parental en el manejo de las situaciones ambientales no estarían relacionadas con el desarrollo de las $\mathrm{AE}^{26}$ aun cuando Kuhle y cols., reportó una niña de 3 años, con múltiples $\mathrm{AE}$ asociadas a crisis y status epilépticos, con exámenes normales, que revirtieron con una terapia neuropsicológica familiar ${ }^{27}$. Creemos recomendable la evaluación psicológica del núcleo familiar para evitar el desarrollo de comorbilidades asociadas a estos eventos traumáticos.

En base a lo anteriormente expuesto, el estudio de la cinética de fierro, un hemograma para evaluar anemia, los estudios electrofisiológicos cardíacos y neurológicos son necesarios para hacer el diagnóstico diferencia en los casos graves ${ }^{15}$.

Entre estos diagnósticos diferenciales mencionamos las apneas centrales, epilepsia y arritmias cardíacas. Las apneas centrales están asociadas al neonato o prematuros y ocurren en el sueño ${ }^{28}$. Una buena historia clínica puede evitar un diagnóstico incorrecto de epilepsia, ya que estas ocurren en sueño y vigilia, sin gatillante emocional, el post-ictal es prolongado, la cianosis ocurre post evento y no antes como en la AE y el EEG frecuentemente es anormal ${ }^{29}$.

Igualmente es necesario hacer el diagnóstico diferencial de una $\mathrm{AE}$ la presencia de patología cardíaca como arritmias cardíacas primarias, síndrome QT largo entre otros ${ }^{7}$.

\section{Conclusiones}

El conocimiento del cuadro clínico, fisiopatología y estudio de diagnósticos diferenciales de las AE es importante para el médico general y pediatra que se ve enfrentado a este tipo de pacientes. Por otra parte, existen alternativas terapéuticas para los casos de AE graves, las cuales han sido expuestas en este documento y deben ser conocidas por el neurólogo pediátrico.

\section{Referencias}

1.- Carman KB, Ekici A, Yimenicioglu S, Arslantas D, Yakut $A$ : Breath holding spells: Point prevalence and associated factors among Turkish children. Pediatr Int 2013; 55 (3): 328-31.

2.- Colina KF, Abelson HT: Resolution of breath-holding spells with treatment of concomitant anemia. J Pediatr 1995; 126 (3): 395-7.

3.- DiMario FJ, Jr: Breath-holding spells in childhood. Am J Dis Child 1992; 146 (1): 125-31.

4.- Lombroso CT, Lerman P: Breathholding spells (cyanotic and pallid infantile syncope). Pediatrics 1967; 39 (4): 563-81.

5.- DiMario FJ, Jr: Prospective study of children with cyanotic and pallid breath-holding spells. Pediatrics 2001; 107 (2): 265-9.

6.- Anil BG, Nedunchezian K, Jayanthini V, Pathmanabhan $M$ : Breath holding spells: evaluation of autonomic nervous system function. Indian Pediatr 2005; 42 (9): 923-7.

7.- Akalin F, Turan S, Guran T, Ayabakan C, Yilmaz Y: Increased QT dispersion in breath-holding spells. Acta Paediatr 2004; 93 (6): 770-4.

8.- Di Pino A, Calabro MP, Gitto P, Bianca I, Oreto G: Permanent cardiac pacing for severe pallid breath-holding spells. Pacing Clin Electrophysiol 2007; 30 (2): 280-2.

9.- Amoozgar H, Saleh F, Farhani N, Rafiei M, Inaloo S, Asadipooya A: Cardiac repolarization changes in the children with breath-holding spells. Iran J Pediatr 2013; 23 (6): 687-92. 
10.- Whitehouse WP: Reflex asytolic syncope. Paediatr Child Health 2013; 23 (6): 263-8.

11.- Southall DP, Samuels MP, Talbert DG: Recurrent cyanotic episodes with severe arterial hypoxaemia and intrapulmonary shunting: a mechanism for sudden death. Arch Dis Child 1990; 65 (9): 953-61.

12.- DiMario FJ Jr, Sarfarazi M: Family pedigree analysis of children with severe breath-holding spells. J Pediatr 1997; 130 (4): 647-51.

13.- Kolkiran A, Tutar E, Atalay S, Deda G, Cin S: Autonomic nervous system functions in children with breath-holding spells and effects of iron deficiency. Acta Paediatr 2005; 94 (9): 1227-31.

14.- Arslan H, Torun E, Akkan JC, Guler S, Bayraktar S: The Evaluation of Physiological and Biochemical Parameters and the Autonomic Nervous Systems of Children with Breath-Holding Spells. Neuropediatrics 2013 Dec 13.

15.- Breningstall GN: Breath-holding spells. Pediatr Neurol 1996; 14 (2): 91-7.

16.- Yilmaz O, Ciftel M, Ozturk K, et al: Assessment of heart rate variability in breath holding children by 24 hour Holter monitoring. Cardiol Young 2013; 19: 1-7.

17.- Carano N, Bo I, Zanetti E, Tchana B, Barbato G, Agnetti $A$ : Glycopyrrolate and theophylline for the treatment of severe pallid breath-holding spells. Pediatrics 2013; 131 (4): e1280-3.

18.- Walsh M, Knilans TK, Anderson JB, Czosek RJ: Successful treatment of pallid breath-holding spells with fluoxetine. Pediatrics 2012; 130 (3): e685-9.

19.- Sartori S, Nosadini M, Leoni L, et al: Pacemaker in complicated and refractory breath-holding spells: When to think about it? Brain Dev 2014; 11.

20.- DiMario FJ Jr, Bauer L, Baxter D: Respiratory sinus arrhythmia in children with severe cyanotic and pallid breath-holding spells. J Child Neurol 1998; 13 (9): 4402.

21.- Zehetner AA, Orr N, Buckmaster A, Williams K, Wheeler $D M$ : Iron supplementation for breath-holding attacks in children. Cochrane Database Syst Rev 2010 12; (5): CD008132.

22.- Orii KE, Kato Z, Osamu F, et al: Changes of autonomic nervous system function in patients with breath-holding spells treated with iron. J Child Neurol 2002; 17 (5): 337-40.

23.- Calik M, Abuhandan M, Aycicek A, Taskin A, Selek $S$, Iscan $A$ : Increased oxidant status in children with breath-holding spells. Child's Nerv Syst 2013; 29 (6): 1015-9.

24.- Sawires H, Botrous O: Double-blind, placebo-controlled trial on the effect of piracetam on breath-holding spells. Eur J Pediatr 2012; 171 (7): 1063-7.

25.- Abbaskhanian A, Ehteshami S, Sajjadi S, Rezai MS: Effects of piracetam on pediatric breath holding spells: a randomized double blind controlled trial. Iran J Child Neurol 2012; 6 (4): 9-15.

26.- Hudaoglu O, Dirik E, Yis U, Kurul S: Parental attitude of mothers, iron deficiency anemia, and breath-holding spells. Pediatr Neurol 2006; 35 (1): 18-20.

27.- Kuhle S, Tiefenthaler M, Seidl R, Hauser E: Prolonged generalized epileptic seizures triggered by breathholding spells. Pediatr Neurol 2000; 23 (3): 271-3.

28.- Instituto de Salud Pública: Apneas emotivas del lactante. Enero 2001; Available at: http://www.saludpublica. com/ampl/ampl10/00d18059.htm. Accedido el 22 junio de 2014.

29.- Mesa T: Patología Pediátrica: Convulsiones en el niño. In: Guiraldes E, Ventura Juncá P, editors. Manual de Pediatría; 2002. p. 18-20. 